\title{
Ultrasound-Guided Lumbar Intradiscal Injection for Discogenic Pain: Technical Innovation and Presentation of Two Cases
}

This article was published in the following Dove Press journal: Journal of Pain Research

\author{
Tsung-Ju Wu $\mathbb{1 D}^{1,2}$ \\ Chen-Yu Hung (iD ${ }^{3}$ \\ Chih-Wei Lee (iD ${ }^{4}$ \\ Stanley Lam (D) \\ Thomas B Clark $\mathbb{D}^{6}$ \\ Ke-Vin Chang ${ }^{3}{ }^{3}$
}

'Graduate Institute of Basic Medical Science, China Medical University, Taichung, Taiwan; ${ }^{2}$ Department of Physical Medicine and Rehabilitation, Yuanlin Christian Hospital, Changhua, Taiwan; ${ }^{3}$ Department of Physical

Medicine and Rehabilitation, National Taiwan University Hospital, Bei-Hu Branch, Taipei, Taiwan; ${ }^{4}$ Department of Medical Imaging, Changhua Christian Hospital, Changhua, Taiwan;

${ }^{5}$ Department of Family Medicine, Faculty of Medicine, The Chinese University of Hong Kong, Hong Kong; ${ }^{6}$ Private Practice Ultrasonographic Training, Vista, CA, USA
Correspondence: Chen-Yu Hung Department of Physical Medicine and Rehabilitation, National Taiwan University Hospital, Bei-Hu Branch, Taipei, Taiwan Email chenyu810@gmail.com

\begin{abstract}
We described two cases and the techniques for using the ultrasound (US) to guide lumbar intradiscal injection with platelet-rich plasma (PRP). The two cases suffered from chronic low back pain. Magnetic resonance imaging revealed posterior annular tear of the L5/S1 intervertebral disc (IVD) in the first case and L4/5 and L5/S1 IVDs in the second case. For the US-guided lumbar intradiscal injection, the patient was placed in a prone position. By placing the transducer in the axial plane at the interlaminar space, the needle was directed toward the center of the aimed IVD. The needle tip was ensured inside the IVD by using the end-feel of sudden reduction of resistance and the poking technique with the transducer oriented in the paramedian sagittal oblique plane. At the follow-up, both patients had significant improvement after the intradiscal PRP injections (visual analogue scale from 7.5 to 1.5 on average). The report indicated US-guided lumbar intradiscal PRP injection to be a feasible approach for treatments of low back pain. Familiarization of the anatomy and sonoanatomy of the lumbar spine is fundamental to achieve the success of intradiscal injection. Keywords: low back pain, lumbar spine, ultrasound, platelet-rich plasma, intervertebral disc
\end{abstract}

\section{Introduction}

The lumbar discogenic pain is one of the most common causes of low back pain. ${ }^{1}$ The diagnosis is made by discography to reproduce the concordant pain. In recent years, intradiscal injection therapy with platelet-rich plasma (PRP) or stem cells has been demonstrated as an effective treatment on these patients. ${ }^{2-4}$ The procedure is mostly performed under fluoroscopy or computed tomography (CT) guidance. ${ }^{3}$ Recently, ultrasound (US) has been an emerging tool utilized in the performance of chronic pain interventions as it is easily available, free of radiation and offers real-time guidance. The common treatment targets of US-guided treatment on the lumbar spine include facet joints, medial branches, and inter-lamina epidural space. ${ }^{5}$ To the best of our knowledge, the US-guided techniques for lumbar intradiscal injection have not been reported. Here, we presented two cases with chronic discogenic low back pain treated with US-guided intradiscal injection of PRP. The approval of institutional review board was waived for retrospective reports of two cases.

\section{Cases}

\section{Case I}

A 37-year-old female patient started to have low back pain since 11 years ago. She denied any trauma history. The pain bothered her life every day and exacerbated in 
long time standing. She had ever received several times of physical therapies, facet joints injections, and acupunctures but the pain did not improve. There was no radiation, numbness, weakness or reflex abnormality. On physical examination, posterior to anterior compression over the L5-S1 interspinous area provoked deep back pain, ${ }^{6}$ and her pain became worse in extension. The magnetic resonance imaging (MRI) revealed a high-intensity zone (HIZ) at the posterior L5-S1 IVD which indicated a posterior annular tear (Figure 1A). We performed PRP (ACP double syringe system, Arthrex, Germany) injection ( $3 \mathrm{~mL})$ to the L5-S1 intervertebral disc (IVD) under US guidance during which the concordant pain was produced (Figure 1B-D, Supplementary Video 1). No procedure-related complications were reported. Three months later, the visual analogue scale (VAS) improved from 8 to 2 and she became more active in physical activities.
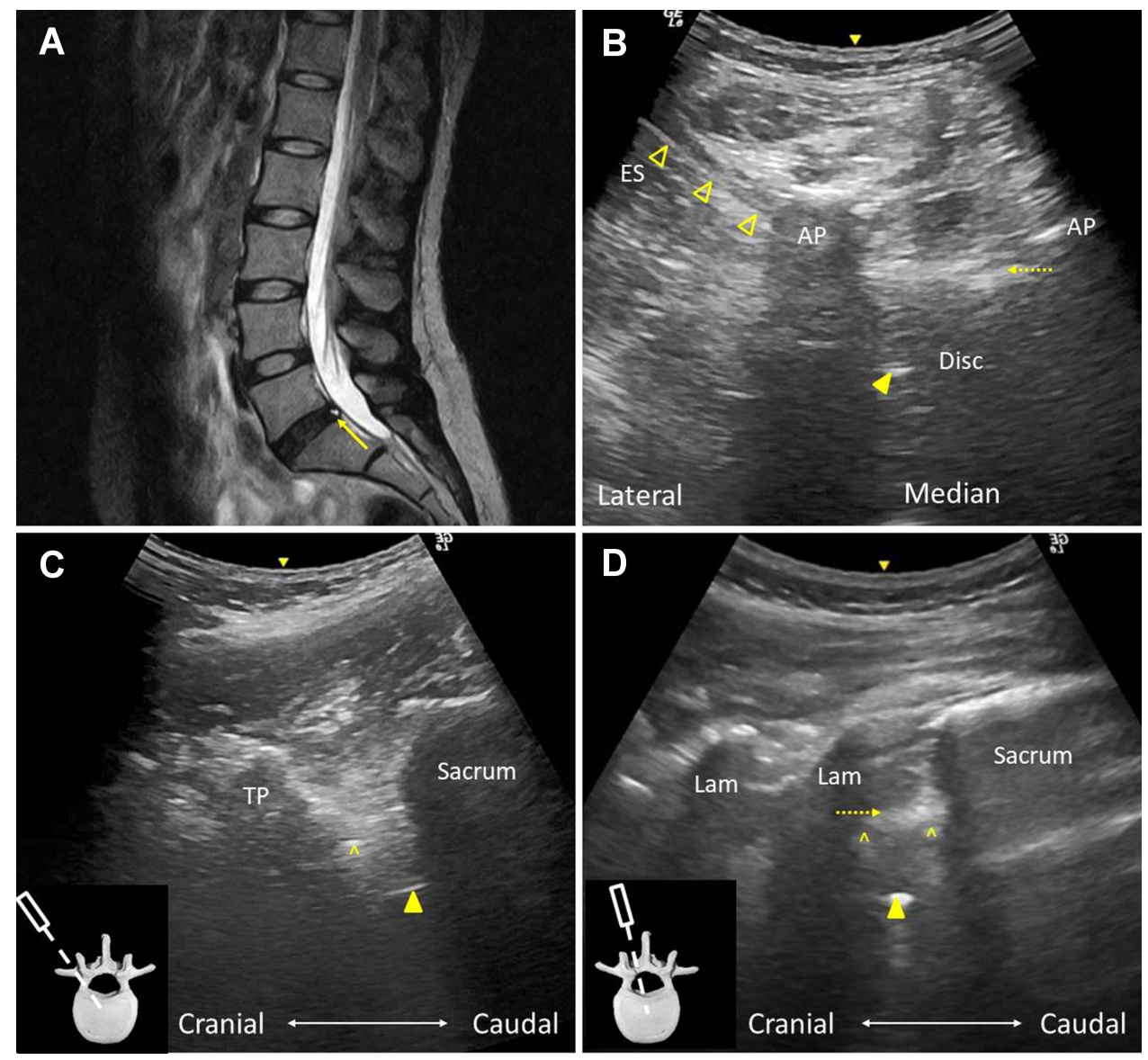

Figure I (A) Sagittal T2-weighted magnetic resonance (MR) imaging demonstrating a high-intensity zone (HIZ, arrow) of the L5/SI intervertebral disc (IVD) at the posterior annulus. (B) Axial ultrasound (US) imaging of the L5/SI interlaminar space showing the in-plane injection of the L5/SI IVD. Note the needle shaft (void arrowheads) was partially blocked by the articular processes. Dashed arrow: posterior longitudinal ligament. Arrowhead: needle tip. (C) Paramedian sagittal oblique US imaging of the lumbar spine with the window between the tip of the transverse process and the superior articular process and (D) the window for interlaminar space showing the needle tip (arrowhead) within the IVD. Note the IVD was situated between the two hyperechoic bony cortex of vertebrae $\left({ }^{\wedge}\right)$. The small inserts illustrate the transducer position and orientation.

Abbreviations: AP, articular process; ES, erector spinae; TP, transverse process; Lam, lamina.

\section{Case 2}

A 36-year-old female patient suffered from chronic low back pain for three years. She was a skateboarder and had ever fallen on the ground for numerous times during skateboarding. She had most obvious pain and stiffness of her low back when she got up in the morning or after a period of immobilization. The pain was centrally located without peripheral radiation. There was no numbness, weakness or reflex abnormality. On physical examination, posterior to anterior compression at the L4-5 and L5-S1 interspinous area provoked deep back pain. The MRI revealed an HIZ at the posterior L4/5 and L5-S1 IVD, respectively (Figure 2A). PRP (ACP double syringe system, Arthrex, Germany) injection $(3 \mathrm{~mL})$ under US guidance was performed to the IVDs which produced the concordant pain (Figure 2B and $\mathrm{C}$, Supplementary Video 2). The patient had no procedurerelated complications. One month later, she reported 

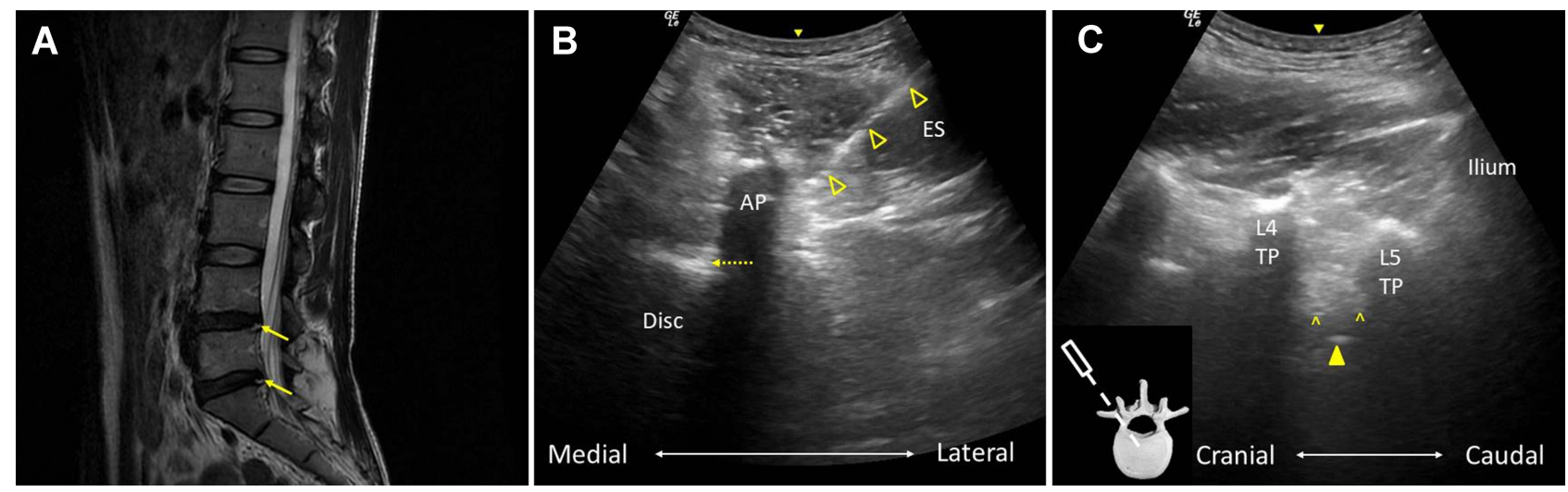

Figure 2 (A) Sagittal T2-weighted magnetic resonance (MR) imaging demonstrating a high-intensity zone (HIZ, arrow) of the L4/5 and L5/SI intervertebral discs (IVDs) at the posterior annulus. (B) Axial ultrasound (US) imaging of the L4/L5 interlaminar space showing the in-plane injection of the L4/L5 IVD. Note the needle tip was blocked by the articular processes. Dashed arrow: posterior longitudinal ligament. Void arrowheads: needle shaft. (C) Paramedian sagittal oblique US imaging of the lumbar spine with the window between the tip of the transverse process and the superior articular process showing the needle tip (arrowhead) within the IVD. Note the IVD was situated between the two hyperechoic bony cortex of vertebrae $\left({ }^{\wedge}\right)$. The small insert illustrates the transducer position and orientation.

Abbreviations: AP, articular process; ES, erector spinae; TP, transverse process.

significant improvement without pain or stiffness when she got up in the morning (VAS improved from 7 to 1 ).

\section{Technique Reports}

For performing the US-guided lumbar IVD injection, the patient was placed in a prone position with one or two pillows underneath the abdomen to reduce the lumbar lordotic curve. Antibiotics (Cefazolin $1 \mathrm{~g}$ ) was injected intravenously before the procedure for infection prophylaxis. Complete aseptic technique was used throughout the procedure. The skin was prepped with chlorhexidine solution and the solution should remain on the skin for at least two minutes to kill the skin bacteria that were usually implicated in the risk of deep tissue infections following skin puncture. ${ }^{7}$ A curvilinear transducer was used for better penetration. Counting of the whole lumbar spine levels was performed under US first to locate the exact level of target and exclude the existence of lumbar sacralization or sacral lumbarization, which could lead to injection to the incorrect level. Then, the transducer was placed between the posterior superior iliac spines for the short axis scanning of the lumbar spine. A "dual-slope roof" appearance was identified first to locate the sacrum. ${ }^{8}$ Then, the transducer was moved cranially until a gap was seen in the middle of the roof, which indicated the L5-S1 interlaminar space. The L4-L5 and the other upper interlaminar spaces were located by sliding the transducer cranially. The lumbar IVD was identified through the interlaminar space in the axial plane. The facet joints were often treated as a landmark to identify the lumbar IVD as the facet joints and the lumbar IVDs were at the same level anatomically.

After the local anesthetics was injected as a skin bleb, a 4-inch needle was injected through the erector spinae muscles under US guidance with an in-plane approach. The concept of the "Kambin triangle" was utilized in which the working triangle bordered by the exiting nerve root, thecal sac, and the caudal vertebral body allowed a safe triangular corridor for access of the dorsolateral IVD (Figure 3). ${ }^{9}$ The trajectory of the needle was pointed to the center of lumbar IVD, passing just under the superior articular process and was slightly cranial to caudal to avoid hitting the nerve root which also courses caudally (Figures 1B and 2B). Once the needle reached the Kambin triangle and the US image of needle was blocked by the bony structures of the superior and inferior articular processes, the transducer was rotated 90 degrees to the paramedian sagittal plane and tilted inward in the window between the tips of the transverse processes and the facet joints to catch the needle tip image using the out-of-plane approach. Care should be taken that the transducer was kept away from the needle entry point to avoid contamination while shifting the transducer position. Then, the needle was advanced slowly until the needle tip reached the annular fibers of lumbar IVD which was located at the lower third between the two adjacent transverse processes (or between the L5 transverse and the sacral alar for L5/S1 IVD) (Figures $1 \mathrm{C}$ and 2C). The entry point of the IVD was identified as there was no hyperechoic bony cortex and the US beam could penetrate to the deeper structures. Also, the end-feel of intradiscal space was soft and elastic when 


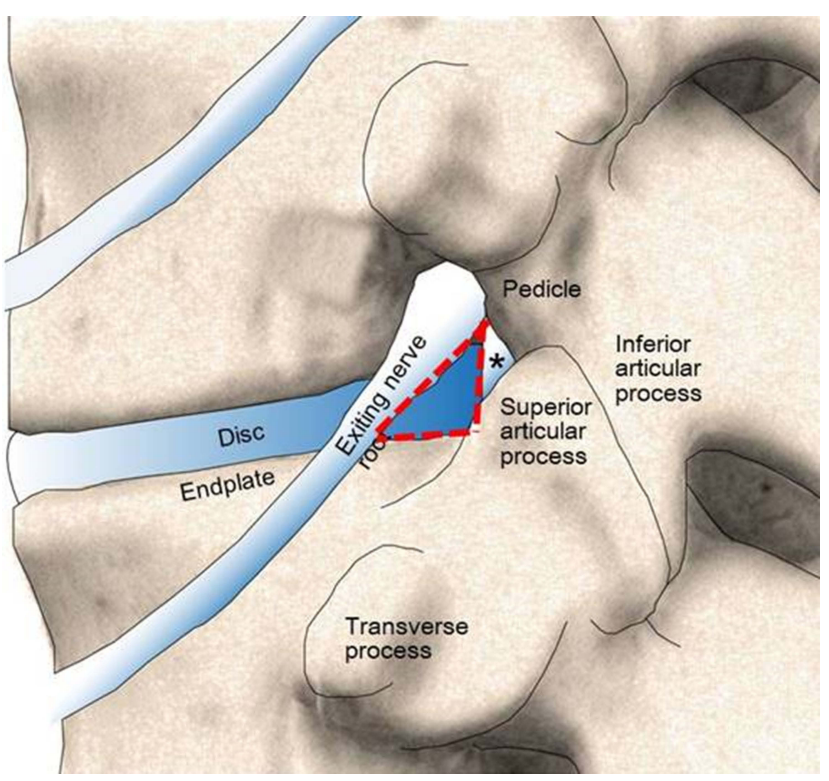

Figure 3 The Kambin triangle (red dashed line) bordered by the exiting nerve root (the hypotenuse), the thecal sac (the asterisk, the height), and the superior border of the caudal vertebra (the width). Note the anatomic relationships of the transverse process, the exiting nerve root, and the intervertebral disc and the facet joint from cranial to caudal.

compared with the hard bony cortex of vertebral body. The power Doppler was used while advancing the needle to avoid radicular artery puncture. The advancement of needle was stopped when the patient felt electric-shock sensation, suggesting irritation of the nerve root by the needle. The needle was redirected caudally as the root was slightly cranial to the IVD level. After the needle tip entered the IVD, the transducer was rotated back to the axial plane of lumbar spine and the needle tip was identified inside the IVD through the interlaminar space by using the poking technique. Another method to confirm whether the needle tip was positioned within the IVD was by placing the transducer in the paramedian sagittal oblique view for the interlaminar space (Figure 1D). The PRP was then injected to both the center and the periphery of the lumbar IVD until great resistance was felt (usually 3-4 mL) and the patient should feel the concordant pain during the injection. After the procedure was finished, the patient was suggested to keep lying on the table for 20 minutes. The patients were suggested to wear the brace immediately following the procedure.

\section{Discussion}

We described the techniques of using real-time US guidance for the lumbar intradiscal injection. Although most of the procedures are performed under fluoroscopy currently, we believe our techniques of US-guided injection can shorten the procedure time and decrease the physical load and radiation exposure. Familiarization of the anatomy and sonoanatomy of the lumbar spine is the foundation to achieve safe and successful intradiscal treatment.

The IVD is avascular in nature with only small metaphyseal arteries around the outer annulus fibrosus. Due to the limited vascular supply and the indirect nutrition access from the adjacent endplate, the IVD has little potential to heal once it is injured. ${ }^{4}$ The pro-inflammatory cytokines produced by the disc cells keep stimulating the outer nociceptors of the annulus fibrosus, leading to the "discogenic pain". ${ }^{3}$ Currently, the main treatments include physical therapy, oral medication, and injection therapy to control the pain. If the conservative treatment fails, surgical intervention with artificial disc or lumbar fusion may be considered although the effectiveness of these surgical procedures is still debated. ${ }^{3}$ PRP is an evolving treatment option that has been widely used in various musculoskeletal injuries. ${ }^{10}$ It involves the preparation of autologous human blood with increased platelet concentration produced by centrifuging of a patient's own blood. The PRP comprises a broad spectrum of growth factors and proteins, hence can stimulate the recruitment, proliferation, and differentiation of cells involved in the healing process. The cytokines, chemokines, and chemokine receptors released by the platelet can regulate the inflammatory and immunological responses of tissue healing. ${ }^{3}$ The fibrins within the PRP may also catalyze sealing of the annular fissure. ${ }^{4}$ Current literature has shown promising results of lumbar intradiscal PRP injection, ${ }^{3,4,11}$ prompting a readily available and easyto-perform imaging tool to guide the PRP delivery.

US has the advantages of zero radiation, real-time continuous visualization of the needle, and the ability to detect neurovascular structures. In the authors' experience, the lumbar intradiscal injection can be performed solely under US guidance in most cases. However, in patients with big body sizes or chronic diseases with paraspinal muscle fatty infiltration, the penetration of US beam energy to the deeper structures can be hindered. Also, in patients with scoliosis, severe spondylotic change, or other spinal deformity, the US image may be very different from the typical appearance. In these difficult cases, US can still serve as a combined guiding tool together with the fluoroscopy to facilitate the procedure. To conclude, this report demonstrated the positive effect of lumbar intradiscal injection of PRP on patients with chronic discogenic low 
back pain and the feasibility of US as an imaging guiding tool for this procedure.

\section{Informed Consent}

The research was performed according to the Declaration of Helsinki principles. We have obtained the written informed consent from the patients for their case details and accompanying images to be published.

\section{Funding}

No funding was received.

\section{Disclosure}

The authors declared no conflict of interest existed.

\section{References}

1. Verrills P, Nowesenitz G, Barnard A. Prevalence and characteristics of discogenic pain in tertiary practice: 223 consecutive cases utilizing lumbar discography. Pain Med. 2015;16:1490-1499.

2. Navani A, Manchikanti L, Albers SL, et al. Responsible, safe, and effective use of biologics in the management of low back pain: American Society of Interventional Pain Physicians (ASIPP) Guidelines. Pain Physician. 2019;22:S1-S74.
3. Mohammed S, Yu J. Platelet-rich plasma injections: an emerging therapy for chronic discogenic low back pain. J Spine Surg. 2018;4:115-122. doi:10.21037/jss.2018.03.04

4. Tuakli-Wosornu YA, Terry A, Boachie-Adjei K, et al. Lumbar intradiskal platelet-rich plasma (PRP) injections: a prospective, double-blind, randomized controlled study. $P M$ R. 2016;8:1-10. doi:10.1016/j.pmrj.2015.08.010

5. Hurdle MF. Ultrasound-guided spinal procedures for pain: a review. Phys Med Rehabil Clin N Am. 2016;27:673-686. doi:10.1016/j. pmr.2016.04.011

6. Nyström B, Weber H, Schillberg B, Taube A. Symptoms and signs possibly indicating segmental, discogenic pain. A fusion study with 18 years of follow-up. Scand J Pain. 2017;16:213-220. doi:10.1016/ j.sjpain.2016.10.007

7. Singh V. The role of cervical discography in interventional pain management. Pain Physician. 2004;7:249-255.

8. Chang KV, Lin CP, Lin CS, et al. Sonographic tracking of trunk nerves: essential for ultrasound-guided pain management and research. J Pain Res. 2017;10:79-88. doi:10.2147/JPR.S123828

9. Tumialan LM, Madhavan K, Godzik J, Wang MY. The history of and controversy over Kambin's triangle: a historical analysis of the lumbar transforaminal corridor for endoscopic and surgical approaches. World Neurosurg. 2019;123:402-408. doi:10.1016/j.wneu.2018.10.221

10. Le ADK, Enweze L, DeBaun MR, Dragoo JL. Current clinical recommendations for use of platelet-rich plasma. Curr Rev Musculoskelet Med. 2018;11:624-634. doi:10.1007/s12178-018-9527-7

11. Cheng J, Santiago KA, Nguyen JT, Solomon JL, Lutz GE. Treatment of symptomatic degenerative intervertebral discs with autologous platelet-rich plasma: follow-up at 5-9 years. Regen Med. 2019;14:831-840. doi:10.2217/rme-2019-0040
Journal of Pain Research

\section{Publish your work in this journal}

The Journal of Pain Research is an international, peer reviewed, open access, online journal that welcomes laboratory and clinical findings in the fields of pain research and the prevention and management of pain. Original research, reviews, symposium reports, hypothesis formation and commentaries are all considered for publication. The manuscript management system is completely online and includes a very quick and fair peer-review system, which is all easy to use. Visit http:// www.dovepress.com/testimonials.php to read real quotes from published authors. 